\title{
O-GIcNAcylation under hypoxic conditions and its effects on the blood-retinal barrier in diabetic retinopathy
}

\author{
CHONG XU, GUODONG LIU, XIAOQIAO LIU and FANG WANG \\ Department of Ophthalmology, Shanghai Tenth People's Hospital Affiliated to \\ Tongji University School of Medicine, Shanghai 200072, P.R. China
}

Received August 25, 2013; Accepted December 12, 2013

DOI: $10.3892 / \mathrm{ijmm} .2013 .1597$

\begin{abstract}
An increase in O-linked N-acetylglucosamine (O-GlcNAc) protein modifications has been observerd in $\mathrm{db} /$ $\mathrm{db}$ mouse retinas. O-GlcNAc-modified proteins in the $\mathrm{db} /$ $\mathrm{db}$ mouse retina have been shown to be localized in the ganglion cell layer, the inner nuclear layer, the retina pigment epithelium (RPE) layer and the inner plexiform layer, in which hypoxia-inducible factor $1 \alpha(\mathrm{HIF} 1 \alpha)$ has also been shown to be localized. In the current study, we examined whether hypoxia increases O-GlcNAcylation in retinal vascular cells under high glucose conditions and whether HIF1 $\alpha$ activation is consistent with the response to and activation of O-GlcNAcylation in retinal lesions in diabetic retinopathy. In addition, the effects of O-GlcNAcylation on the blood-retinal barrier were verified in vitro by the inhibition of O-GlcNAcylation. A time-dependent increase in the O-GlcNAcylation in bovine retinal vascular endothelial cells (BRVECs) was observed following incubation of the cells with high glucose medium (glucose $4.5 \mathrm{~g} / \mathrm{l})$ under hypoxic $\left(1-3 \% \mathrm{O}_{2}\right)$ conditions. Hypoxiainduced BRVEC O-GlcNAcylation was not observed when the BRVECs were transfected with siRNA targeting O-GlcNAc transferase (OGT) or treated with alloxan (an OGT inhibitor) prior to exposure to high glucose. The increase in BRVEC O-GlcNAcylation induced by high glucose, as well as by thiamet G [an O-GlcNAcase (OGA) inhibitor] led to a reduction in occludin expression levels in vitro, which was prevented by treatment with OGT siRNA and alloxan. In conclusion, the current study demonstrates the relationship between O-GlcNAc glycosylation and hypoxia during diabetic retinopathy and that hyperglycemia induced $\mathrm{O}_{2}$ consumption activates HIF1 $\alpha$ and $\mathrm{O}-\mathrm{GlcNAc}$ modification protein in the same retinal layer. The
\end{abstract}

Correspondence to: Dr Fang Wang, Department of Ophthalmology, Shanghai Tenth People's Hospital Affiliated to Tongji University School of Medicine, 301 Middle Yan Chang Road, Shanghai 200072, P.R. China

E-mail: dreyemilwang_122@msn.com

Key words: O-GlcNAcylation, hypoxia, occludin, blood-retinal barrier, diabetic retinopathy reduced protein BRVEC O-GlcNAcylation levels exert protective effects on the blood-retinal barrier and thus represent a potential therapeutic target for the treatment of diabetic retinopathy.

\section{Introduction}

In 1984, Torres and Hart found that serine and threonine residues in nuclear and cytoplasmic proteins can undergo O-linked $\mathrm{N}$-acetylglucosamine (O-GlcNAc) modification (1). The difference with various other modifications, is that O-GlcNAc modification is regulated by a single pair of enzymes: the glycosyltransferase, O-GlcNAc transferase (OGT) (EC 2.4.1.94; GI: 6006036), and the glycosidase, O-GlcNAcase (OGA) (EC .3.2.1.52; GI: 13646137). There are $>1,000$ types of proteins that can be O-GlcNAcylated. O-GlcNAcylation affects protein interactions, cellular localization, protein degradation and participates in the regulation of various biological functions (2). The role of O-GlcNAc modification in type 2 diabetes, neurological degenerative diseases, as well as its role in cardiovascular and cerebrovascular diseases has been investigated in depth and is becoming a key factor in regulating cellular processes (3).

Certain studies have revealed that O-GlcNAc modification mediates diabetic vascular dysfunction (4). Increased O-GlcNAcylation has been shown to enhance reactivity to constrictor stimuli (5); O-GlcNAcylation has also been shown to enhance vascular contraction contribute through the activation of the endothelin-1 (ET-1) or RhoA kinase pathway (6).

Although O-GlcNAcylation and diabetes mellitus (DM) have been extensively studied in the cardiovascular and neuroendocrine fields, reports on the alterations that occur in retinal O-GlcNAcylation levels in diabetic retinopathy are limited. More precisely, the location of the retinal cells which are affected by protein O-GlcNAcylation under hypoxic conditions, as well as the effects of O-GlcNAcylation on the blood retinal barrier, have not been reported. In the current study, we examined whether hypoxia increases O-GlcNAcylation in retinal vascular cells under high glucose conditions and whether hypoxia-inducible factor $1 \alpha$ (HIF $1 \alpha$ ) activation is consistent with the response to and activation of O-GlcNAcylation in retinal lesions in diabetic retinopathy. In addition, the effects of O-GlcNAcylation on the blood-retinal barrier were verified in vitro by the inhibition of O-GlcNAcylation. 
An adequate supply of oxygen and nutrients is critical to retinal function. Prolonged hypoxia induced by high glucose can induced changes in the retina. Any changes that occur in oxygen or glucose levels can lead to cellular damage at the molecular level. Elucidating these mechanisms will not only enhance our understaning of the pathogenesis of diabetic retinopathy, but may also lead to the development of novel therapeutic strategies (7).

\section{Materials and methods}

Reagents. The reagents purchased were as follows: HIF1 $\alpha$ antibody (Bethyl Laboratories, Inc., Montgomery, TX, USA); CTD110.6 antibody (Cell Signaling Technology, Inc., Beverly, MA, USA); OGT antibody (Santa Cruz Biotechnology, Inc., Santa Cruz, CA, USA); anti-von Willebrand Factor antibody (Abcam, Cambridge, MA, USA; VWF, ab6994); Alexa Fluor 488-labeled goat anti-Rabbit IgG and occludin (Abcam); 4',6'-diamidino-2-phenylindole hydrochloride (DAPI) and heparin (Sigma, St. Louis, MO, USA); Dulbecco's modified Eagle's medium (DMEM; high glucose medium, $4.5 \mathrm{~g} / 1$ glucose and low glucose medium, 1 g/l glucose) (Gibco, Carlsbad, CA, USA); MEM, HEPES, collagenase type IV, DNase, Pronase, thiamet G, alloxan, AG490 and bovine serum albumin (BSA) (all obtained from Sigma); fetal calf serum (FBS) TrypsineEDTA (Invitrogen, Carlsbad, CA, USA); extracellular matrix (ECM), ECGS (100X), penicillin, streptomycin, ascorbic acid, and amphotericin-B (ScienCell, Carlsbad, CA, USA); plastic tissue culture flask (Costar, Cambridge, MA, USA); and Immobilon-NC Transfer Membrane (Millipore, Billerica, MA, USA). Leica confocal laser scanning microscope (Leica Microsystems, Mannheim, Germany) was used for scanning. The $\mathrm{db} / \mathrm{db}$ mice and $\mathrm{db} / \mathrm{m}$ mice were purchased from SLRC Laboratory Animal Centre of Shanghai Institutes for Biological Sciences.

Isolation of bovine retinal vascular endothelial cells (BRVECs) and primary cell culture. The isolation of BRVECs was performed according to a modified method originally developed by Banumathi et al (8). Briefly, the freshly isolated retinas from bovine eyes were washed with ice-cold $\mathrm{CO}_{2}$-independent medium, $100 \mathrm{U} / \mathrm{ml}$ penicillin and $100 \mu \mathrm{g} / \mathrm{ml}$ streptomycin. In a laminar flow hood, the retinas were washed with the same solution, homogenized on ice 6 times and centrifuged at $400 \mathrm{x} \mathrm{g}$ for $10 \mathrm{~min}$ at $4^{\circ} \mathrm{C}$. The pelleted retinal tissue was resuspended in $10 \mathrm{ml}$ of serum-free MEM. The retinal sections were transferred to a tube containing $4 \mathrm{ml}$ of an enzyme cocktail which consisted of $500 \mu \mathrm{g} / \mathrm{ml}$ collagenase type IV, $200 \mu \mathrm{g} / \mathrm{ml}$ DNase and $200 \mu \mathrm{g} / \mathrm{ml}$ pronase in $10 \mathrm{mM}$ phosphate-buffered saline (PBS) containing $0.5 \%$ BSA. The retinal sections along with the enzyme cocktail were incubated at $37^{\circ} \mathrm{C}$ for $30 \mathrm{~min}$. The pellet was passed through a $53-\mu \mathrm{m}$ steel mesh. The trapped blood vessels were removed with the use of sterile forceps and then washed 3 times with cold MEM by centrifugation at $400 \mathrm{x}$ g for $5 \mathrm{~min}$. The pellet containing microvessel fragments was suspended in DMEM supplemented with $10 \% \mathrm{FBS}, 90 \mu \mathrm{g} / \mathrm{ml}$ heparin, $5 \mu \mathrm{g} / \mathrm{ml}$ ascorbic acid, ECGS $100 \mathrm{U} / \mathrm{ml}$ penicillin-G, $100 \mu \mathrm{g} / \mathrm{ml}$ streptomycin and $2.5 \mu \mathrm{g} / \mathrm{ml}$ amphotericin-B. The cells were then incubated at $37^{\circ} \mathrm{C}$ in a $5 \% \mathrm{CO}_{2}$ incubator. The culture medium was replaced every 3 days and after reaching confluence, the cells were passaged with a $0.25 \%$ solution of trypsin-EDTA. von Willebrand factor (vWF) and CD31 antibodies were used for the identification of BRVECs. The BRVECs were then cultured under high and low glucose conditions for $72 \mathrm{~h}$ and were starved in serum-free DMEM for a further $24 \mathrm{~h}$. The cells were then treated with chemicals (thiamet G or OGT siRNA) for $24 \mathrm{~h}$. In the hypoxia group, the cells were cultured in low and high glucose medium, and were then exposed to a culture chamber containing $1-3 \% \mathrm{O}_{2}$ for $2,6,12,24$ and $48 \mathrm{~h}$.

Immunofluorescence staining of BRVECs for $v W F$. The BRVECs were fixed overnight in $4 \%$ paraformaldehyde, then washed 3 times with PBS for $5 \mathrm{~min}$. They were pre-treated with $0.5 \% \mathrm{H}_{2} \mathrm{O}_{2}$-methanol and then blocked and permeabilized by incubation in $5 \%$ goat serum containing $0.1 \%$ Triton X-100 and 3\% BSA. After having been washed with PBS, the cells were incubated overnight with the primary $\mathrm{vWF}$ antibody, followed by incubation with the Alexa Fluor 488-conjugated secondary antibody in PBS solution for $1 \mathrm{~h}$ at room temperature. The cells were then washed several times with PBS and incubated for $10 \mathrm{~min}$ with DAPI. After having been washed extensively with PBS, the stained cells were examined under a Leica immunofluorescence microscope.

siRNA tansfection and treatment with thiamet $G$. Total protein O-GlcNAcylation was inhibited with the use of OGT siRNA or alloxan and was enhanced with the use of thiamet G. siRNA targeting OGT and the negative control siRNA were purchased from GenePharma (Shanghai, China). siRNA (ON-TARGETplus SMARTpool; $100 \mathrm{nM}$ ) was incubated with Lipofectamine 2000 (Invitrogen) in $1.0 \mathrm{ml}$ of serum-free medium for $30 \mathrm{~min}$. The siRNA-Lipofectamine 2000 complex was then added to the cells in $4.0 \mathrm{ml}$ of serum-free medium and maintained for days. On the 3rd day, the cells were incubated with high glucose for $48 \mathrm{~h}$. The other cell groups were incubated with thiamet $\mathrm{G} 48 \mathrm{~h}$ followed by high glucose medium.

Treatment with AG490. The BRVECs were treated with $80 \mu \mathrm{M}$ AG490 for $24 \mathrm{~h}$, then cell lysis was prepared for western blot analysis. AG490, a Janus kinase 2 (JAK2) inhibitor, can reduce the phosphorylation of JAK2, as a protective factor of the retinal barrier. Cells that were treated with AG490 were used as the positive control. AG490 can not only reduce the expression of VEGF, but can also increase the expression of occludin.

Western blot analysis. Cell lysates and the retinal tissues from $\mathrm{db} / \mathrm{db}$ and $\mathrm{db} / \mathrm{m}$ mice were treated with lysis buffer $[10 \mathrm{mM}$ Tris (pH 7.5), $150 \mathrm{mM} \mathrm{NaCl}, 5 \mathrm{mM}$ EDTA, $1 \%$ Triton X-100, $1 \mathrm{mM}$ DTT, $0.1 \mathrm{mM}$ PMSF, $10 \%$ glycerol and a protease inhibitor cocktail tablet] for $40 \mathrm{~min}$ on ice. The retinal tissues were homogenized in lysis buffer followed by centrifugation at $13,500 \mathrm{rpm}$ for $10 \mathrm{~min}$. The total protein concentration was calculated using the Enhanced BCA protein assay kit (Beyotime Institute of Biotechnology, Haimen, China). Lysate protein $(30 \mu \mathrm{g})$ was subjected to $4-10 \%$ SDS-PAGE and electrophoretically transferred onto a nitrocellulose (NC) membrane. After blotting, the membrane was blocked in 5\% fat-free dry milk for $1 \mathrm{~h}$ and then incubated with the specific primary anti- 
body overnight at $4^{\circ} \mathrm{C}$. Protein bands were detected with the use of an enhanced chemiluminescence (ECL) detection kit (Amersham) following hybridization with the HRP-conjugated secondary antibody or with the use of LI-COR Odyssey infrared laser imaging system in order to detect the scanned image. Densitometric analysis was performed using Image J software (version 1.43, Broken Symmetry Software, Bethesda, MD, USA). For each experiment, the measurements were repeated 3 times.

Experimental animals and treatment. We used C57BLKS/J $\mathrm{db} / \mathrm{db}$ mice as an animal model of type 2 diabetes, while $\mathrm{C} 57 \mathrm{BLKS} / \mathrm{J} \mathrm{db} / \mathrm{m}$ mice were selected as the control group. Male C57BLKS/J db/db ( $\mathrm{n}=20,10$ weeks old) and $\mathrm{db} / \mathrm{m}$ mice $(\mathrm{n}=10$, 10 weeks old) were purchased from the SLRC Laboratory Animal Centre of Shanghai Institutes for Biological Sciences. They were housed in cages in a constant environment (room temperature, $20-22^{\circ} \mathrm{C}$; room humidity, $40-60 \%$ ) with a $12 \mathrm{~h} / 12 \mathrm{~h}$ light/dark cycle. Blood glucose levels remained at 19.8-29.8 mM. All procedures were approved by the Animal Ethics Committee of Tongji University, Shanghai, China. The $\mathrm{db} / \mathrm{db}$ mice were divided into 2 groups: an early-stage diabetic retinopathy group (DM, 12-16 weeks, $\mathrm{n}=10$ ) and a late-stage diabetic retinopathy group (DM, 24-32 weeks, $n=10$ ). The eyes were immediately enucleated, and the retinas were then dissected. Retinal tissues were kept at $-80^{\circ} \mathrm{C}$ until further analysis.

Immunofluorescence staining of mouse retinas. The eyes from the $\mathrm{db} / \mathrm{db}$ mice with varying stages of diabetic retinopathy (12, 24 and 32 weeks) and from the control $\mathrm{db} / \mathrm{m}$ mice were enucleated and embedded with optimal cutting temperature (OCT) compound and then frozen at $-80^{\circ} \mathrm{C}$. The $10-\mathrm{mm}$-thick frozen sections were fixed in $4 \%$ paraformaldehyde overnight. Following pre-treatment with $0.5 \% \mathrm{H}_{2} \mathrm{O}_{2}$-methanol for $30 \mathrm{~min}$ and PBS containing 5\% normal goat serum, $0.5 \%$ BSA and $0.1 \%$ Triton $\mathrm{X}-100$ for $30 \mathrm{~min}$ at room temperature, the sections were incubated overnight at $4^{\circ} \mathrm{C}$ with anti-O-GlcNAc [antimouse CTD 110.6 (1:100), anti-OGT antibody (1:100)] and anti-HIF1 $\alpha(1: 100)$ antibodies. The sections were then rinsed with PBS and incubated at room temperature for $1 \mathrm{~h}$ with Alexa Fluor 488-conjugated secondary antibody. All antibodies were diluted in PBS containing $0.5 \%$ goat serum, $0.5 \%$ BSA, and $0.1 \%$ Triton $\mathrm{X}-100$. Instead of a primary antibody, goat serum was used as the negative control. The sections were examined with a Leica confocal laser scanning microscope. Images were captured and processed.

In situ hybridization of HIFl $\alpha$ of mouse retinas. The antisense and sense oligonucleotide probes (Boster Biological Technology Co., Wuhan, China) were designed according to the mouse HIF-1a transcript sequences: i) 5'-TTATGAGCTT GCTCATCAGTTGCCACTTCC-3'; ii) 5'-CTCAGTTTGAAC TAACTGGACACAGTGTGT-3'; iii) 5'-GGCCGCTCAATTT ATGAATATTATCATGCT-3'.

The tissue sections were fixed at room temperature for 20-30 min in 4\% paraformaldehyde in 0.1 M PBS (PH 7.4), containing 1/1,000 diethylpyrocarbonate DEPC. Endogenous peroxidase and biotin were blocked with $30 \% \mathrm{H}_{2} \mathrm{O}_{2}$-methanol (1:50) for $30 \mathrm{~min}$. The sections were then permeabilized with proteinase K (Catalog no. S3020; Dako Denmark A/S,
Glostrup, Denmark) and washed 3 times with Tris-buffered saline. The sections were fixed again with the aforementioned solution. Biotinylated probes designed for HIF1 $\alpha$ were mixed in hybridization buffer ( $50 \%$ formamide) at a concentration of $200 \mathrm{ng} / \mathrm{ml}$. Probes were added to the sections, which were then covered with coverslips and heated at $92^{\circ} \mathrm{C}$ for $5 \mathrm{~min}$. Hybridization was carried out in a humidified chamber at $37^{\circ} \mathrm{C}$ overnight. The sections were washed in $2 \mathrm{X}$ saline-sodium citrate (SSC) for $10 \mathrm{~min}$ followed by 3 washes in Tris-buffered saline and then blocked at $37^{\circ} \mathrm{C}$ for 30 min with blocking solution. The sections were incubated with biotinylated mouse anti-digoxin solution at $37^{\circ} \mathrm{C}$ for $60 \mathrm{~min}$, then washed 4 times with PBS. For hybridization signal detection, SABC and DAB kits were used according to the manufacturer's instructions (Boster Biological Technology Co.).

Statistical analysis. Statistical analyses were performed using SPSS software (version 13.0; SPSS, Chicago, IL, USA). The unpaired Student's t-test was used to assess the significance between 2 groups. One-way ANOVA was used to compare 3 or more groups, while the Mann-Whitney U test or Rank Cases method were used to examine the heterogeneity of variance. A P-value $<0.05$ was considered to indicate a statistically significant difference.

\section{Results}

Characteristics of BRVECs. The cells initially grew as capillary-like structures and demonstrated the typical cobblestone morphology of endothelial cells at confluence (Fig. 1B and C). These cells were characterized as vascular endothelial cells by VWF antigen expression. Using immunofluorescence, the positive expression of vWF antigen in the BRVECs was demonstrated by green particles in the cytoplasm (Fig. 1A and B). BSA staining was used as a negative control (Fig. 1E) while bovine retinal vascular tissue immunostaining was used as a positive control (Fig. 1D).

Total protein O-GlcNAcylation and HIFla retinal distribution in db/db mice with varying stages of diabetic retinopathy. Protein O-GlcNAcylation in the retinas of $\mathrm{db} / \mathrm{db}$ mice was detected by CTD110.6 immunofluorescence and presented a distribution pattern consistent with HIF1 $\alpha$ distribution as found by immunofluorescence and in situ hybridization (Fig. 2). $\mathrm{O}-$ GlcNAcylation appeared in the retinal ganglion cell, inner nuclear and retina pigment epithelium (RPE) layers which are first affected by diabetic retinopathy (Fig. 2B and D), and consequently in the inner plexiform layer (Fig. 2F and $\mathrm{H}$ ). HIF1 $\alpha$ (Fig. 2J and G) and CTD110.6 expression levels (Fig. 2L) were observed at almost undetectable levels in the $\mathrm{db} / \mathrm{m}$ mouse retinas (negative controls).

$O$-GlcNAcylation levels in vivo and in vitro. The protein expression of O-GlcNAc was determined by western blot analysis using CTD110.6 and OGT antibodies as described in Materials and methods. O-GlcNAcylation was increased in the retinas of 14- and 24-week-old db/db mice compared to those of the age-matched control mice (Fig. 3A). A similar increase was observed in O-GlcNAcylation in BRVECs cultured under high glucose conditions (Fig. 3B). In addition, culture under 
A

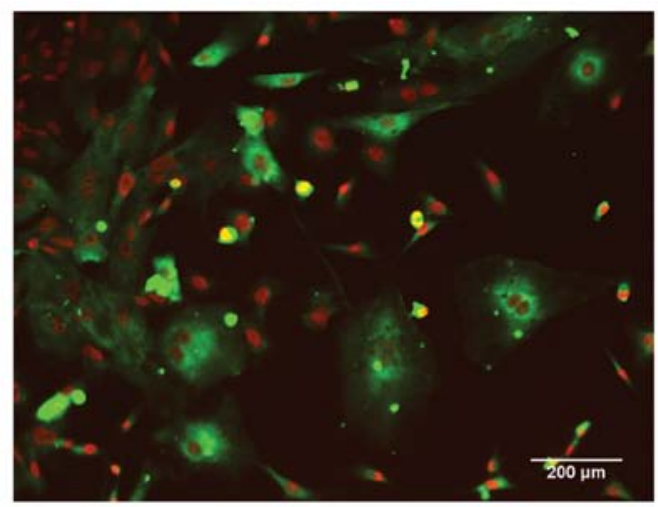

B

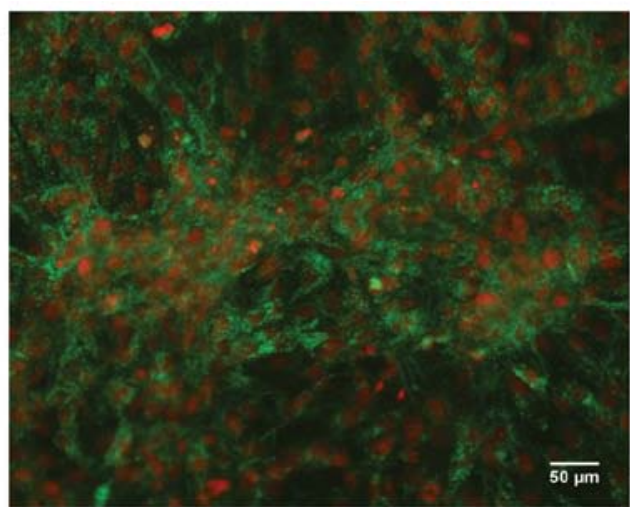

C
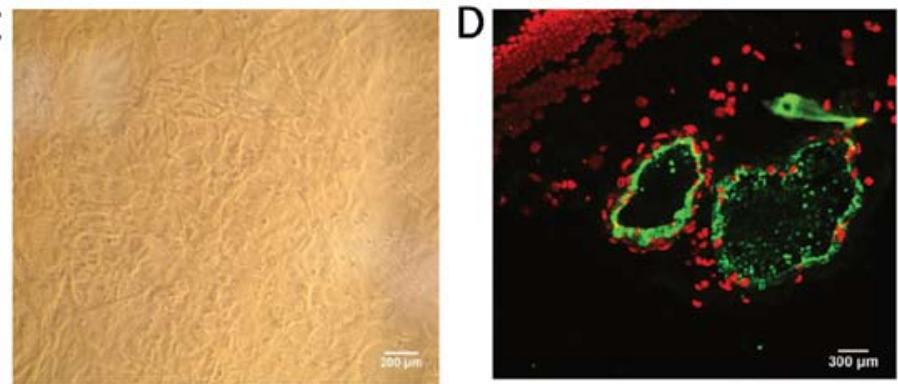

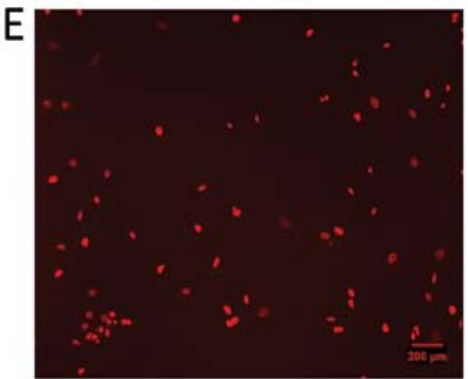

Figure 1. (A and C) Normal cultured bovine retinal vascular endothelial cells (BRVECs). (A and B) Immunostaining: BRVECs were incubated with vWF antibody. (B and C) Cultured BRVECs formed tube-like structures. (D) Bovine retinal vessel tissue was used as a positive control group. (E) Goat serum was used as a negative control group with 5\% BSA instead of primary antibody incubation.

hypoxic conditions induced a time-dependent increase in O-GlcNAcylation (Fig. 3C).

Changes in O-GlcNAcylation levels in BRVECs and the bloodretinal barrier. OGT siRNA inhibition of O-GlcNAcylation in BRVECs in under a high glucose state (Fig. 4A) led to an increased expression of occludin and to reduced VEGF expression levels (Fig. 4C), while increased O-GlcNAcylation was observed following treatment with thiamet $\mathrm{G}$ (Fig. 4B and C). These results indicate that inhibiting O-GlcNAcylation in BRVECs may protect the blood-retinal barrier and reduce VEGF expression.

\section{Discussion}

Diabetic retinopathy is a serious complication of diabetes, leading to blindness in individuals with diabetes (9). O-GlcNAc signaling participates in the pathogenesis of diabetes (10-13). Recent studies have suggested that it also participates in the pathogenesis of diabetic retinopathy (14). The elevated expression of O-GlcNAc has emerged as a regulator of the cellular stress response (15). One of the major causes of cellular stress is hypoxia $(16,17)$ and it is associated with the development of diabetic retinopathy $(18,19)$. In other studies, when multiple cell lines were subjected to diverse types of stress (including hypoxia), there was a rapid and global increase in O-GlcNAc expression levels (20). Hypoxia upregulates the expression of the epitope $\mathrm{H}$ containing an $\mathrm{O}-\mathrm{GlcNAc}$ residue in human ependymal cells (21). Increased OGT levels enhance $\mathrm{O}-\mathrm{GlcN}$ Acylation and reduce cardiomyocyte death following hypoxia (22). O-GlcNAcylation levels have been shown to increase in cardiac cells folloiwng exposure to hypoxia for $4 \mathrm{~h}$ and early reoxygenation; this was confirmed in a follow-up study by the same group (23).

In the current study, it was demonstrated that the O-GlcNAc expression levels increased in vitro and in vivo not only in a high glucose state but also under hypoxic conditions. BRVEC $\mathrm{O}-$ GlcNAcylation in a high glucose state was exacerbated by hypoxia. In diabetic retinopathy, the reduced expression levels of occludin represent a decline in blood-retinal barrier function. OGT siRNA, which inhibits O-GlcNAcylation can protect the blood-retinal barrier by increasing the expression levels of occludin in BRVECs in vitro under high glucose conditions. Thus, it is reported herein that O-GlcNAc signaling, which is associated with HIF1 $\alpha$, is one of the regulatory factors of blood-retinal barrier function in the pathogenesis of diabetic retinopathy.

The possible mechanisms involved in triggering the increase in O-GlcNAcylation under hypoxic conditions are the following: Firstly, O-GlcNAc-mediated cytoprotection, as well as hypoxia which causes endoplasmic reticulum (ER) stress, have been shown to be involved in the pathogenesis of diabetes (24-26). The enhancement of O-GlcNAcylation has been shown to exert cytoprotective effects during hypoxia, ischemia and oxidative stress (27-29). The mitochondria are critical targets of O-GlcNAc-mediated cytoprotection $(22,27,29,30)$. Moreover, O-GlcNAc signaling can directly attenuate oxidative stress-induced cellular dysfunction (27) and calcium overload (29,31). Further studies are required in order to gain a better understanding of diabetic retinopathy. 

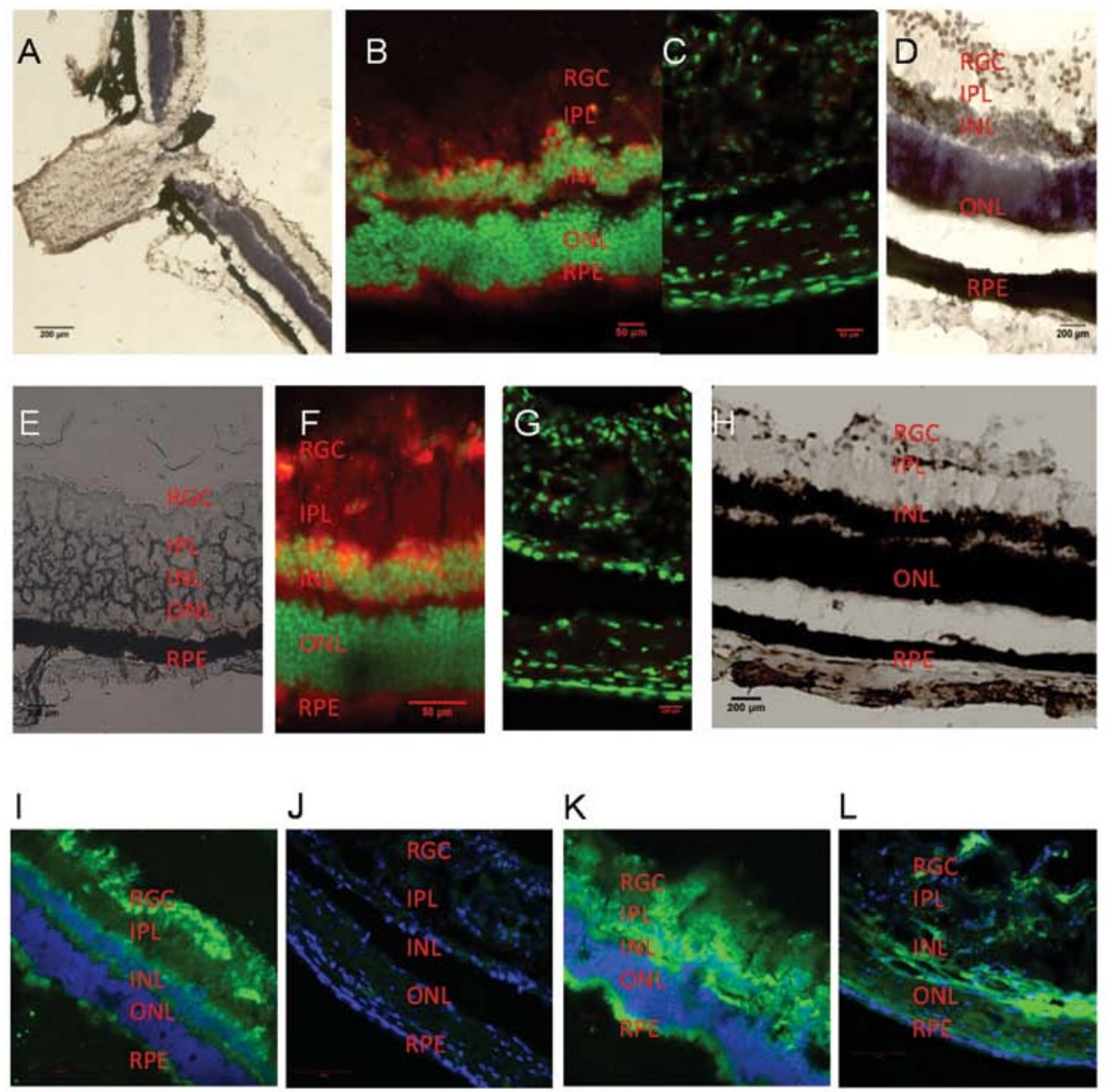

L
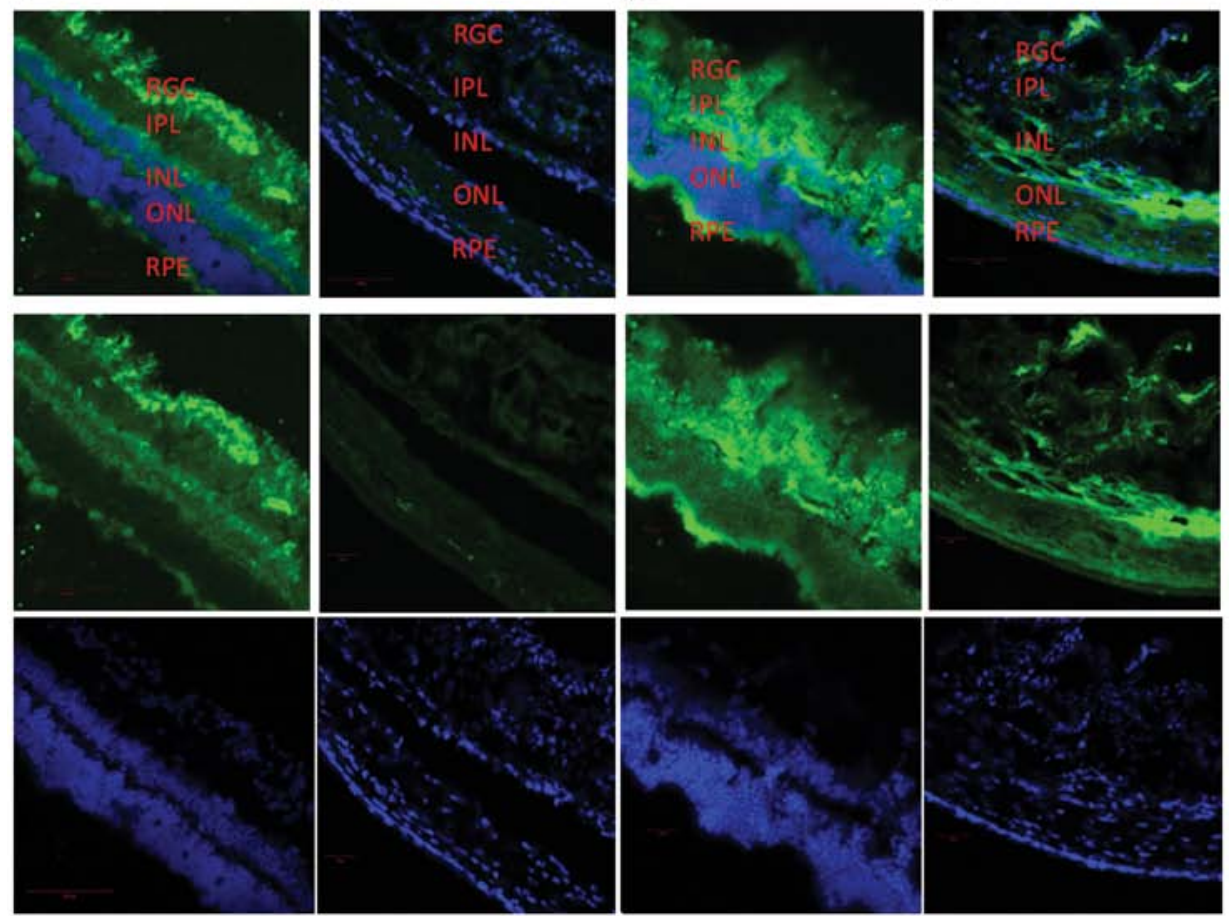

Figure 2. (A) HIF1 $\alpha$ in situ hybridization of db/db mouse retinas (magnification, x100). (B) HIF1 $\alpha$ immunofluorescence of retinas of 12-week-old db/db mice (magnification, x200). HIF1 $\alpha$ is stained red and is expressed mainly in the retinal ganglion cell (RGC) layer, the inner nuclear layer (INL) and the retinal pigment epithelium (RPE) layer. The outer nuclear layer (ONL) shows negative staining. (C) HIF1 $\alpha$ immunofluorescence of 12 -week-old db/m mouse retinas of the control group, (magnification, x400). (D) HIFla in situ hybridization of the retinas of 12 -week-old db/db mice (magnification, $\mathrm{x} 400$ ) present the same distribution of immunostaining. (E) H\&E staining of db/db mouse retinas. (F) HIF1 $\alpha$ immunofluorescence of 32-week-old db/db mouse retinas (magnification, $\mathrm{x} 400$ ). HIF1 $\alpha$ is stained red and appears mainly in the RGC layer, the INL, the inner plexiform layer (IPL) and the RPE layer. The ONL shows negative staining (G) HIF1 $\alpha$ immunofluorescence of 32-week-old db/m mouse retinas of the control group (magnification, $\mathrm{x} 400$ ). (H) HIF1 $\alpha$ in situ hybridization of the retinas of 32-week-old db/db mice (magnification, x200); brown shows the positive staining. (I) HIF1 $\alpha$ immunofluorescence of 24-week-old db/db mouse retinas (magnification, x200). HIF1 $\alpha$ is expressed mainly in the RGC, the INL, the IPL and the RPE layer. (K) Retinal CTD110.6 immunofluorescence of 24-week-old db/db mice (magnification, $x 200$ ), presented a positive staining pattern similar to that of HIF1 $\alpha$. Mainly the RGC, the INL, the RPE layer and the IPL were stained. ( $\mathrm{J}$ and L) Retinal HIF1 $\alpha$ (magnification, x200, J) and CTD110.6 (magnification, x200, L) immunofluorescence of the control group db/m mice. Low HIF1 $\alpha$ and CTD110.6 expression levels were observed.

Secondly, it has been demonstrated that the hexosamine signaling pathway (HSP) is linked to O-GlcNAc cycling and plays an important role in nutrient sensing (32). HSP expres- sion represents an ubiquitous molecular mechanism which prevents the deleterious effects caused by stress (33), while O-GlcNAcylation plays an important role in regulating DNA 
A
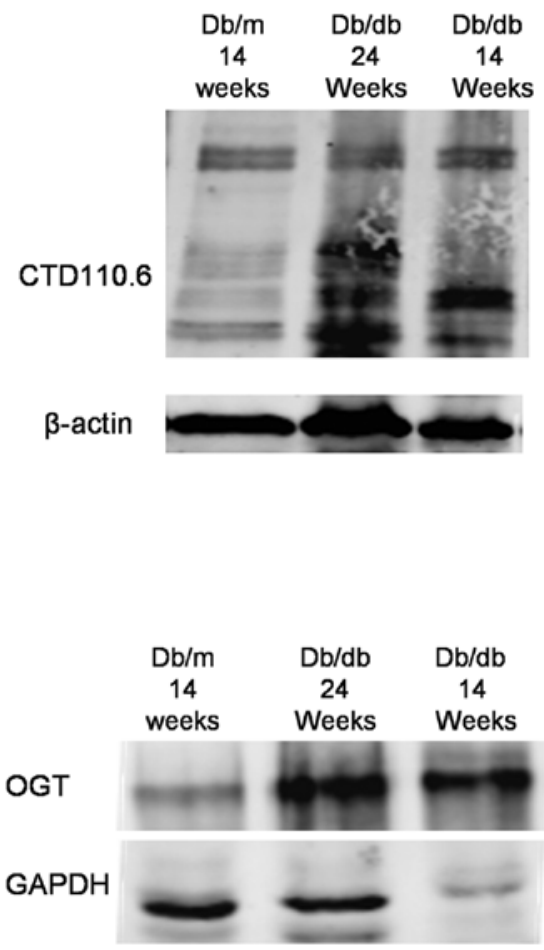

B
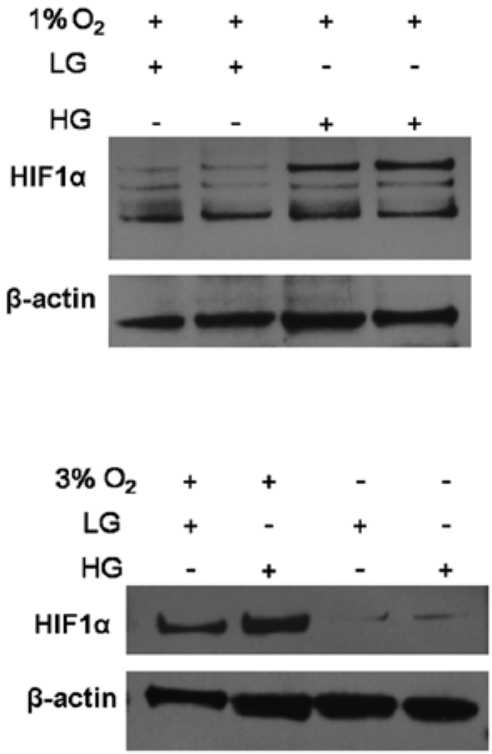

C

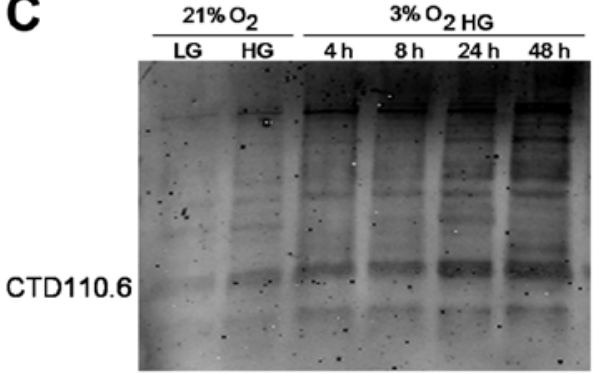

$\beta$-actin $\rightarrow-\mathrm{H}$
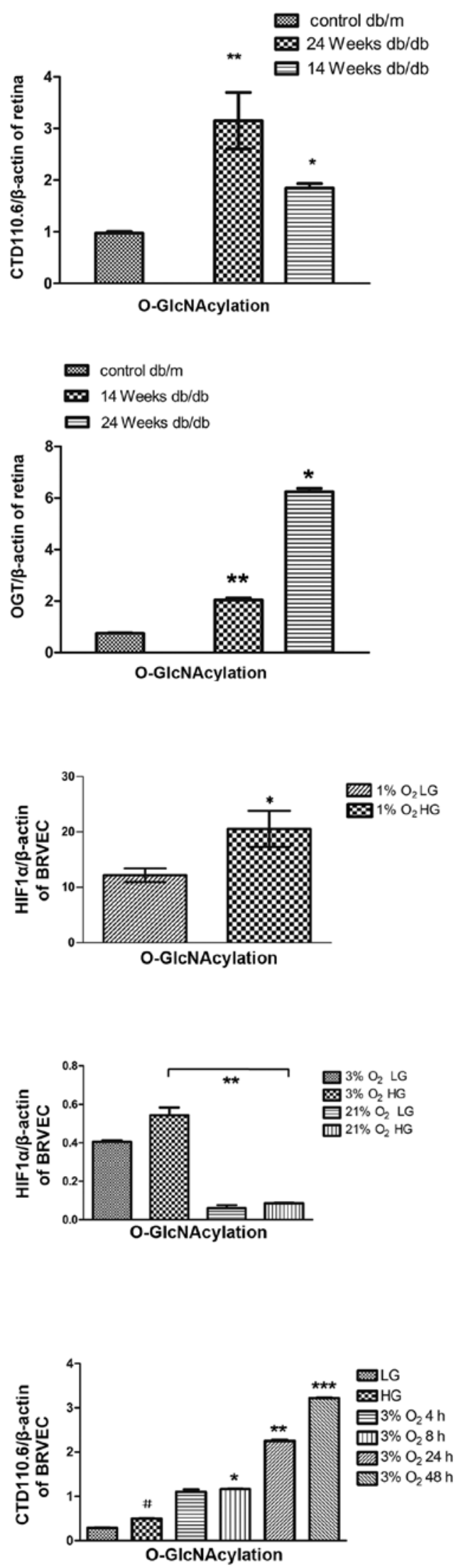

Figure 3. (A) The protein expression levels of CTD110.6 and OGT (O-GlcNAcylation) in the retinas of db/db mice were markedly increased compared with those of the control group $\left({ }^{*} \mathrm{P}<0.05,{ }^{* *} \mathrm{P}<0.01\right)$. (B) HIF1 $\alpha$ expression levels in the high glucose group (HG) were higher under hypoxic conditions than those in the low glucose group (LG) $\left({ }^{*} \mathrm{P}<0.05\right)$. The western blots and graph depict the elevated expression levels of HIF1 $\alpha$ when in vitro oxygen levels are low $\left({ }^{* * *} \mathrm{P}<0.01\right)$. (C) High glucose increases CTD110.6 expression levels, and O-GlcNAcylation, in bovine retina vascular endothelial cells (BRVECs) $\left({ }^{\mathrm{P}} \mathrm{P}<0.05\right)$. CTD110.6 expression levels in BRVECs cultured under high glucose conditions. present a time-dependent increase which is due to hypoxia $\left({ }^{* * * *, * * * *} \mathrm{P}<0.05\right)$. 
A
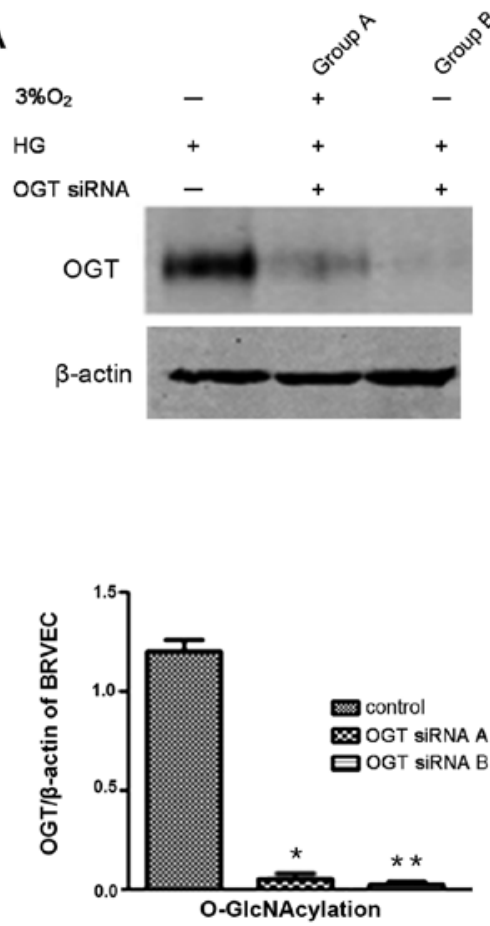

B

Thiamet $\mathrm{G} 5 \mu \mathrm{M}$
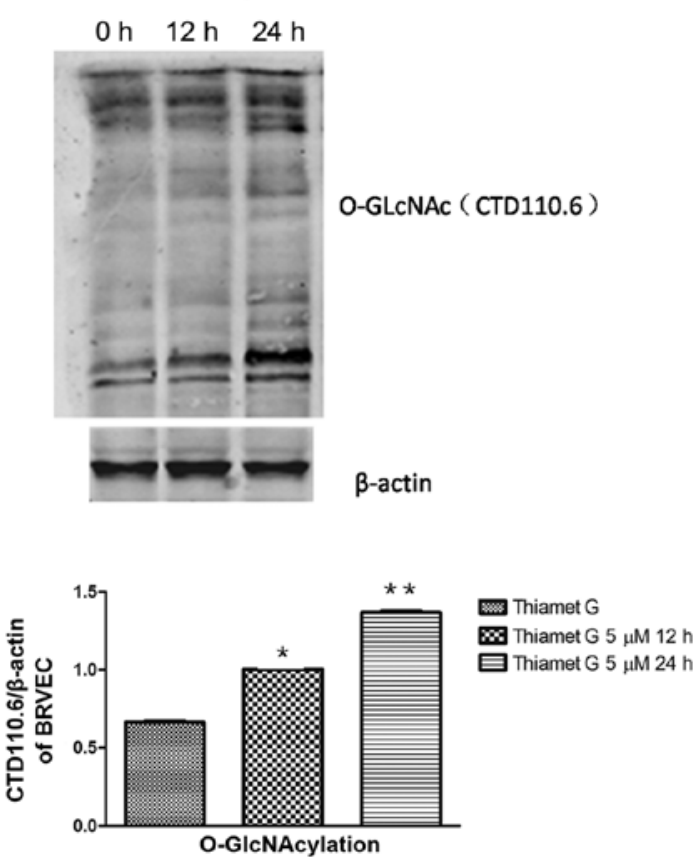

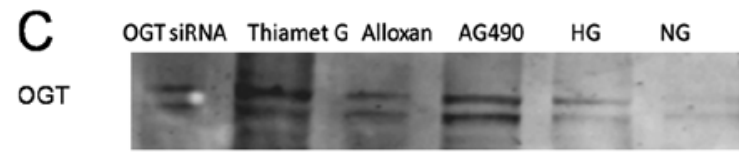

Occludin

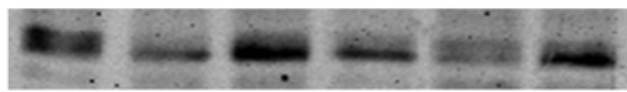

VEGF

$\beta$-actin
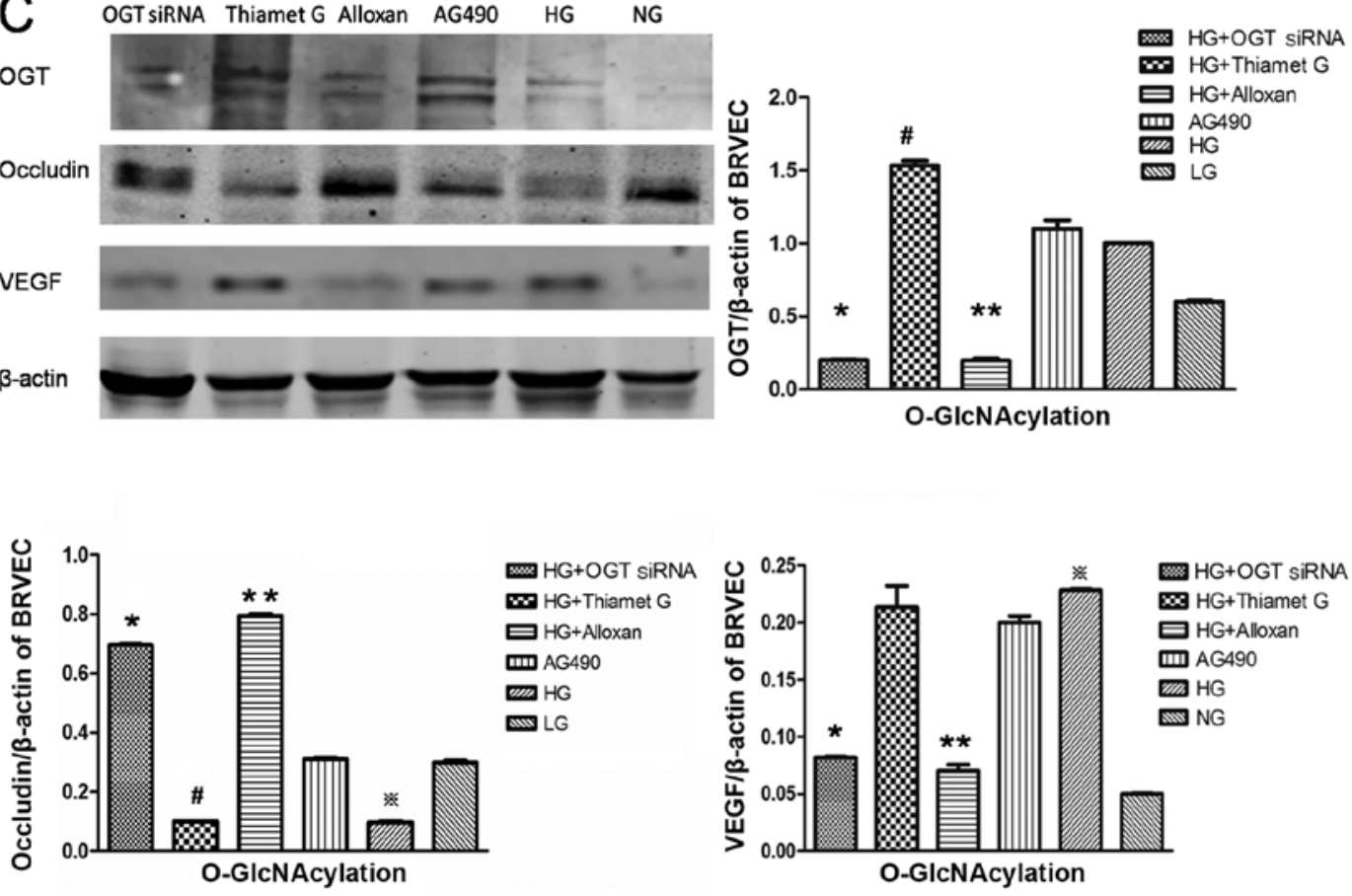

Figure 4. (A) OGT siRNA inhibition of O-GlcNAcylation in bovine retina vascular endothelial cells (BRVECs) in 3\% $\mathrm{O}_{2}$ (OGT siRNA group A) and high glucose (HG, OGT siRNA group B). Very low expression levels of OGT (O-GlcNAcylation) were observed in the OGT siRNA groups (P<0.05). (B) Increase in O-GlcNAcylation in BRVECs following treatment with thiamet $\mathrm{G} 5 \mu \mathrm{M}$ at 12 and $24 \mathrm{~h}$ under high glucose conditions. According to the graph and the western blot, a gradual increase in O-GlcNAcylation (CTD110.6 antibody) was observed. (C) In a high glucose state occludin protein expression was decreased in BRVECs $\left({ }^{*} \mathrm{P}<0.05\right)$, but increased following the administration of alloxan (inhibitor of O-GlcNAcylation) or OGT siRNA $\left({ }^{*},{ }^{* *} \mathrm{P}<0.05\right)$. Thiamet $\mathrm{G}$ enhancement of O-GlcNAcylation leads to reduced expression levels of occludin ( $\left.{ }^{*} \mathrm{P}<0.05\right)$ in BRVECs, while AG490 (a STAT3 inhibitor) on the other hand leads to the opposite result, in other words to increased occludin expression levels. High glucose levels lead to increased expression of VEGF in BRVECs, which can be reduced by inhibiting $\mathrm{O}-$ GlcNAcylation with OGT siRNA or alloxan $\left({ }^{* *}, *{ }^{*} \mathrm{P}<0.05\right)$.

damage or repair through signaling pathways following stress (15).

It is still unclear as to the exact changes that occur in O-GlcNAcylation levels in the diabetic retina. In the present study, we aimed to determine which retinal layer is affected first and to a greater extent by O-GlcNAcylation during diabetic retinopathy in $\mathrm{db} / \mathrm{db}$ mice.

The results of immunofluorescence and in situ hybridization (Fig. 2) revealed that the distribution of the O-GlcNAcylated factors is consistent with HIF1 $\alpha$ distribution in the $\mathrm{db} / \mathrm{db}$ 
mouse retina. During the early stages of diabetic retinopathy (12 weeks), protein O-GlcNAcylation is distributed in the retinal ganglion cell layer, inner nuclear layer and RPE layer. During the later stages of the disease ( 24 and 32 weeks), it is not only distributed in the aforementioned layers but also in the inner plexiform layer. During the early stages of diabetic retinopathy, high glucose levels lead to retina hypoxia (34). According to a recent study, HIF1 $\alpha$ expression levels have been shown to increase significantly in the vitreous fluid of surgically-treated eyes with proliferative diabetic retinopathy (PDR) (35), which also verifies the existence of hypoxia throughout the entire duration of diabetes. The data reported herein demostrate that hypoxia is accompanied by protein O-GlcNAcylation, a finding that reveals that oxygen is necessary for O-GlcNAcylation of the retinal protein.

The photoreceptors of the inner layer, the outer plexiform layer and the deeper regions of the inner plexiform layer are considered dominant oxygen consumers of the rat retina (36). The higher oxygen demand of these layers including the 'OFF' pathway ganglion cells which are normally located in the deeper inner plexiform layer, may render them more sensitive to hypoxia (36).

In the hyperoxia state, such as respired $100 \%$ oxygen, oxygen consumption of the inner retinal layer exceeds that of the photoreceptor layer (37-39). Studies have also shown that VEGF mRNA expression levels in the retina of diabetic rats are significantly increased, mainly in the retinal ganglion cell layer and the inner nuclear layer (40). These data are iin accordance with our observation that HIF1 $\alpha$ is distributed first in the inner nuclear layer, retinal ganglion cell layer and RPE layer, and then in the inner plexiform layer in $\mathrm{db} / \mathrm{db}$ mouse retinas. HIF1 $\alpha$ is a molecular level oxygen sensor and represents the degree of hypoxia (41). Hyperglycemia-induced $\mathrm{O}_{2}$ consumption activates HIF1 $\alpha$ and other hypoxia-associated genes (42). Hypoxia induces a decrease in adenosine triphosphate (ATP) levels, ion imbalance and free radicals which cause ganglion cell, inner nuclear layer cell and RPE cell apoptosis (43). The distribution of O-GlcNAc is consistent with the distribution of HIF1 $\alpha$ in the $\mathrm{db} / \mathrm{db}$ mouse retina and reveals that the change in retinal $\mathrm{O}-$ GlcNAcylation levels is mainly influenced by the location where hypoxia occurs. This may be a compensatory response which leads to tissue damage.

In this study, we also investigated the decreased expression levels of occludin in cultured BRVECs under high glucose conditions; these levels increased following transfection with OGT siRNA. Further studies are required to confirm whether this change occurs due to the direct regulation of occludin phosphorylation, or to other kinase-mediated signal transduction pathways.

Occludin is known as a transmembrane component of tight junctions (TJs) and together with ZO-1, constitute the TJ between cells (44). It has also been confirmed that occludin plays a role in the blood-retinal barrier breakdown during diabetic retinopathy $(45,46)$. Under hypoxic conditions, the transient increase in intracellular $\mathrm{Ca}^{2+}$ activates extracellular signal-related kinases, such as calmodulin-dependent kinase, triggering the cascade reaction, causing changes in the mRNA levels and protein expression of occludin and other TJ-associated proteins, eventually leading to cell barrier function damage (47).
It is more evident when occludin is phosphorylated. Occludin serine/threonine phosphorylation aggregates in the TJ location, while fewer occludin phosphorylated residues are distributed in the cytoplasm of the basement membrane side. The increased serine/threonine phosphorylation of occludin can enhance the barrier function (48). Factors, such as lack of calcium, phorbol (phorbol ester) stimulation (44) and pathological conditions (45), that destroy TJs, can change the state of occludin phosphorylation, which influences TJ barrier functions (46). It is well known that O-GlcNAc modification due to glycosylation and phosphorylation, is a complementary adjustment, which functions in a regulatory mode, as suppressing glycosylated occludin expression may enhance its phosphorylation (1), leading to the increase of TJ barrier functions. There exists a novel interplay between occludin and O- $\beta$-glycosylation (49). Due to the complexity of the measurement methods, further studies arerequired to confirm whether occludin itself exerts O-GlcNAcylation.

The present study demonstrates that during the course of diabetic retinopathy, the degree of retinal hypoxia becomes more severe and O-GlcNAcylation is closely associated with hypoxia in the retinas of $\mathrm{db} / \mathrm{db}$ mice with type 2 diabetes. Protein O-GlcNAcylation appears mainly first in the ganglion cell layer, the inner nuclear layer and the RPE layer and at a later stage, in the inner plexiform layer. In addition, we found that the inhibition of BRVEC O-GlcNAcylation under high glucose conditions can prevent the increase in occludin and the decrease in VEGF expression levels, and may thus contribute to the protection of blood-retinal barrier in diabetic retinopathy. Further studies are required to gain a better understanding of the molecular basis of O-GlcNAcylation which plays a role in the pathogenesis of diabetic retinopathy.

\section{Acknowledgements}

This study was supported in part by the project of Shen Kang Hospital Development Center (SHDC12010207, Shanghai, China). We are grateful for the assistance of Dr Bebee and Dr Yingbo Shui of the Research Centre of Ophthalmology at Washington University (USA).

\section{References}

1. Torres CR and Hart GW: Topography and polypeptide distribution of terminal $\mathrm{N}$-acetylglucosamine residues on the surfaces of intact lymphocytes. Evidence for O-linked GlcNAc. J Biol Chem 259: 3308-3317, 1984.

2. Hart GW, Housley MP and Slawson C: Cycling of O-linked beta-N-acetylglucosamine on nucleocytoplasmic proteins. Nature 446: 1017-1022, 2007.

3. Lima VV, Rigsby CS, Hardy DM, Webb RC and Tostes RC: O-GlcNAcylation: a novel post-translational mechanism to alter vascular cellular signaling in health and disease: focus on hypertension. J Am Soc Hypertens 3: 374-387, 2009.

4. Lima VV, Spitler K, Choi H, Webb RC and Tostes RC: $\mathrm{O}-$ GlcNAcylation and oxidation of proteins: is signalling in the cardiovascular system becoming sweeter? Clin Sci (Lond) 123: 473-486, 2012.

5. Lima VV, Giachini FR, Carneiro FS, et al: Increased vascular O-GlcNAcylation augments reactivity to constrictor stimuli - VASOACTIVE PEPTIDE SYMPOSIUM. J Am Soc Hypertens 2: 410-417, 2008.

6. Kim do H, Seok YM, Kim IK, Lee IK, Jeong SY and Jeoung NH: Glucosamine increases vascular contraction through activation of RhoA/Rho kinase pathway in isolated rat aorta. BMB Rep 44: 415-420, 2011. 
7. Grimm C and Willmann G: Hypoxia in the eye: a two-sided coin High Alt Med Biol 13: 169-175, 2012.

8. Banumathi E, Haribalaganesh R, Babu SS, Kumar NS and Sangiliyandi G: High-yielding enzymatic method for isolation and culture of microvascular endothelial cells from bovine retinal blood vessels. Microvasc Res 77: 377-381, 2009.

9. AliTK andEl-Remessy AB: Diabetic retinopathy: current management and experimental therapeutic targets. Pharmacotherapy 29 : 182-192, 2009.

10. Ruan HB, Singh JP, Li MD, Wu J and Yang X: Cracking the O-GlcNAc code in metabolism. Trends Endocrinol Metab 24: 301-309, 2013.

11. Jensen RV,Zachara NE, Nielsen PH, Kimose HH, Kristiansen SB and Botker HE: Impact of O-GlcNAc on cardioprotection by remote ischaemic preconditioning in non-diabetic and diabetic patients. Cardiovasc Res 97: 369-378, 2013.

12. Bennett CE, Johnsen VL, Shearer J and Belke DD: Exercise training mitigates aberrant cardiac protein O-GlcNAcylation in streptozotocin-induced diabetic mice. Life Sci 92: 657-663, 2013

13. McLarty JL, Marsh SA and Chatham JC: Post-translational protein modification by $\mathrm{O}$-linked $\mathrm{N}$-acetyl-glucosamine: its role in mediating the adverse effects of diabetes on the heart. Life Sci 92: 621-627, 2013.

14. Gurel Z, Sieg KM, Shallow KD, Sorenson CM and Sheibani N: Retinal O-linked $\mathrm{N}$-acetylglucosamine protein modifications: implications for postnatal retinal vascularization and the pathogenesis of diabetic retinopathy. Mol Vis 19: 1047-1059, 2013.

15. Zachara NE, Molina H, Wong KY, Pandey A and Hart GW: The dynamic stress-induced 'O-GlcNAc-ome' highlights functions for O-GlcNAc in regulating DNA damage/repair and other cellular pathways. Amino Acids 40: 793-808, 2011.

16. Tai TC, Wong-Faull DC, Claycomb R and Wong DL: Hypoxic stress-induced changes in adrenergic function: role of HIF1 alpha. J Neurochem 109: 513-524, 2009.

17. Witt KA, Mark KS, Huber J and Davis TP: Hypoxia-inducible factor and nuclear factor kappa-B activation in blood-brain barrier endothelium under hypoxic/reoxygenation stress. J Neurochem 92: 203-214, 2005.

18. Arden GB and Sivaprasad S: Hypoxia and oxidative stress in the causation of diabetic retinopathy. Curr Diabetes Rev 7: 291-304, 2011.

19. Li C, Chen P, Zhang J, et al: Enzyme-induced vitreolysis can alleviate the progression of diabetic retinopathy through the HIF-1alpha pathway. Invest Ophthalmol Vis Sci 54: 4964-4970, 2013.

20. Zachara NE, O'Donnell N, Cheung WD, Mercer JJ, Marth JD and Hart GW: Dynamic O-GlcNAc modification of nucleocytoplasmic proteins in response to stress. A survival response of mammalian cells. J Biol Chem 279: 30133-30142, 2004.

21. Arvanitis LD, Vassiou K, Kotrotsios A and Sgantzos MN: Hypoxia upregulates the expression of the $\mathrm{O}$-linked $\mathrm{N}$-acetylglucosamine containing epitope $\mathrm{H}$ in human ependymal cells. Pathol Res Pract 207: 91-96, 2011.

22. Ngoh GA, Watson LJ, Facundo HT, Dillmann W and Jones SP: Non-canonical glycosyltransferase modulates post-hypoxic cardiac myocyte death and mitochondrial permeability transition. J Mol Cell Cardiol 45: 313-325, 2008.

23. Ngoh GA, Facundo HT, Zafir A and Jones SP: O-GlcNAc signaling in the cardiovascular system. Circ Res 107: 171-185, 2010.

24. Araki E, Oyadomari S and Mori M: Endoplasmic reticulum stress and diabetes mellitus. Intern Med 42: 7-14, 2003.

25. Harding HP, Zeng H, Zhang Y, et al: Diabetes mellitus and exocrine pancreatic dysfunction in perk $^{-/}$mice reveals a role for translational control in secretory cell survival. Mol Cell 7: $1153-1163,2001$

26. Ozcan U, Cao Q, Yilmaz E, et al: Endoplasmic reticulum stress links obesity, insulin action, and type 2 diabetes. Science 306 : 457-461, 2004.

27. Jones SP, Zachara NE, Ngoh GA, et al: Cardioprotection by $\mathrm{N}$-acetylglucosamine linkage to cellular proteins. Circulation 117: $1172-1182,2008$

28. Liu J, Marchase RB and Chatham JC: Increased O-GlcNAc levels during reperfusion lead to improved functional recovery and reduced calpain proteolysis. Am J Physiol Heart Circ Physiol 293: H1391-H1399, 2007.
29. Ngoh GA, Facundo HT, Hamid T, Dillmann W, Zachara NE and Jones SP: Unique hexosaminidase reduces metabolic survival signal and sensitizes cardiac myocytes to hypoxia/reoxygenation injury. Circ Res 104: 41-49, 2009.

30. Champattanachai V,Marchase RB and Chatham JC: Glucosamine protects neonatal cardiomyocytes from ischemia-reperfusion injury via increased protein O-GlcNAc and increased mitochondrial Bcl-2. Am J Physiol Cell Physiol 294: C1509-C1520, 2008.

31. Ngoh GA, Hamid T, Prabhu SD and Jones SP: O-GlcNAc signaling attenuates ER stress-induced cardiomyocyte death. Am J Physiol Heart Circ Physiol 297: H1711-H1719, 2009.

32. Hanover JA, Krause MW and Love DC: The hexosamine signaling pathway: O-GlcNAc cycling in feast or famine. Biochim Biophys Acta 1800: 80-95, 2010.

33. Guinez C, Mir AM, Leroy Y, Cacan R, Michalski JC and Lefebvre T: Hsp70-GlcNAc-binding activity is released by stress, proteasome inhibition, and protein misfolding. Biochem Biophys Res Commun 361: 414-420, 2007.

34. Kennedy A and Frank RN: The influence of glucose concentration and hypoxia on VEGF secretion by cultured retinal cells. Curr Eye Res 36: 168-177, 2011.

35. Loukovaara S, Koivunen P, Ingles M, Escobar J, Vento $M$ and Andersson S: Elevated protein carbonyl and HIF-1alpha levels in eyes with proliferative diabetic retinopathy. Acta Ophthalmol: May 29, 2013 (Epub ahead of print).

36. Yu DY and Cringle SJ: Oxygen distribution and consumption within the retina in vascularised and avascular retinas and in animal models of retinal disease. Prog Retin Eye Res 20: 175-208, 2001.

37. Yu DY, Cringle SJ, Yu PK and Su EN: Intraretinal oxygen distribution and consumption during retinal artery occlusion and graded hyperoxic ventilation in the rat. Invest Ophthalmol Vis Sci 48: 2290-2296, 2007.

38. Cringle SJ and Yu DY: A multi-layer model of retinal oxygen supply and consumption helps explain the muted rise in inner retinal $\mathrm{PO}(2)$ during systemic hyperoxia. Comp Biochem Physiol A Mol Integr Physiol 132: 61-66, 2002.

39. Yu DY, Cringle SJ and Su EN: Intraretinal oxygen distribution in the monkey retina and the response to systemic hyperoxia. Invest Ophthalmol Vis Sci 46: 4728-4733, 2005.

40. Sandercoe TM, Geller SF, Hendrickson AE, Stone J and Provis JM: VEGF expression by ganglion cells in central retina before formation of the foveal depression in monkey retina: evidence of developmental hypoxia. J Comp Neurol 462: 42-54, 2003.

41. Semenza GL: Hydroxylation of HIF-1: oxygen sensing at the molecular level. Physiology (Bethesda) 19: 176-182, 2004.

42. Bensellam M, Duvillie B, Rybachuk G, et al: Glucose-induced $\mathrm{O}(2)$ consumption activates hypoxia inducible factors 1 and 2 in rat insulin-secreting pancreatic beta-cells. PLoS One 7: e29807, 2012.

43. Yang L, Tan $\mathrm{P}, \mathrm{Zhou} \mathrm{W}$, et al: $\mathrm{N}$-acetylcysteine protects against hypoxia mimetic-induced autophagy by targeting the HIF-1 alpha pathway in retinal ganglion cells. Cell Mol Neurobiol 32: 1275-1285, 2012

44. Feldman GJ, Mullin JM and Ryan MP: Occludin: structure, function and regulation. Adv Drug Deliv Rev 57: 883-917, 2005.

45. Cummins PM: Occludin: one protein, many forms. Mol Cell Biol 32: 242-250, 2012.

46. Dorfel MJ and Huber O: Modulation of tight junction structure and function by kinases and phosphatases targeting occludin. J Biomed Biotechnol 2012: 807356, 2012.

47. Clarke H, Soler AP and Mullin JM: Protein kinase C activation leads to dephosphorylation of occludin and tight junction permeability increase in LLC-PK1 epithelial cell sheets. J Cell Sci 113 (Pt 18): 3187-3196, 2000.

48. Simonovic I, Arpin M, Koutsouris A, Falk-Krzesinski HJ and Hecht G: Enteropathogenic Escherichia coli activates ezrin, which participates in disruption of tight junction barrier function. Infect Immun 69: 5679-5688, 2001.

49. Butt AM, Feng D, Nasrullah I, et al: Computational identification of interplay between phosphorylation and O-beta-glycosylation of human occludin as potential mechanism to impair hepatitis $\mathrm{C}$ virus entry. Infect Genet Evol 12: 1235-1245, 2012. 